Symptomatic COVID-19 was more frequent in patients with a history of DAH, RPGN and hlgG. hlgG during the follow-up was significantly associated with COVID-19 in multivariable analysis $(p=0.01$, OR=20,6 \%95 Cl (2-210). Comparison of patients who died of COVID-19 and survived showed that female sex, PNS involvement and hlgG during the clinical course and hospital admission were risk factors for increased mortality (Table 1). Age, smoking, treatments, history of flares or serious infections, remission status and chronic renal insufficiency did not differ between groups.

Conclusion: The frequency and mortality from COVID-19 is found to be high in our AAV cohort compared to previous reports (1). Patients with serious lung or renal involvement are prone to symptomatic COVID-19. Previously reported severe outcomes on RTX therapy might be related to consequent hlgG. High dose IVIG treatment may not be sufficient in improving survival in AAV patients with severe COVID-19 and hlgG.

REFERENCES:

[1] Severity of COVID-19 and survival in patients with rheumatic and inflammatory diseases: data from the French RMD COVID-19 cohort of 694 patients. 2020:annrheumdis-2020-218310.

Disclosure of Interests: None declared

DOI: 10.1136/annrheumdis-2021-eular.3880

\section{POS1258 $\quad$ MISSING THE WINDOW OF OPPORTUNITY: EARLY ARTHRITIS CLINICS IN TIMES OF COVID-19}

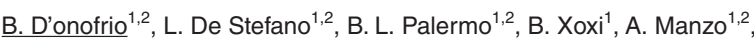
C. Montecucco ${ }^{1,2}$, S. Bugatti ${ }^{1,2} .{ }^{1} I R C C S$ Policlinico San Matteo Foundation, Division of Rheumatology, Pavia, Italy; ${ }^{2}$ University of Pavia, Department of Internal Medicine and Therapeutics, Pavia, Italy

Background: The outcomes of patients with chronic inflammatory arthritis (IA), such as rheumatoid arthritis (RA), have dramatically improved over the past 20 years. Earlier identification of IA and prompter treatment institution have been key advancements, promoted by the constitution of Early Arthritis Clinics (EAC) and the development of more sensitive classification criteria for RA. The outbreak of new COronaVIrus Disease 2019 (COVID-19) has quickly become a global health emergency and has forced a rearrangement in the management of other non-COVID-19 diseases. The impact of the lock-down of the healthcare systems on chronic inflammatory diseases such as RA is expected to be significant but is at present unknown.

Objectives: To assess the effects of the lock-down imposed by the COVID-19 pandemic on the referral and clinical presentation of patients with new-onset RA. Methods: Data were retrieved from the Pavia EAC inception cohort, established in 2005 for the early identification of patients with new-onset IA. Referral criteria to the EAC include: $\geq 3$ swollen joints (SJ) and/or $<3$ SJ and positive squeeze test and/or $<3 \mathrm{SJ}$ and morning stiffness $>30 \mathrm{~min}$. Demographic and clinical characteristics of the patients are assessed at baseline and regularly over follow-up. At 31 Dec 2020, the Pavia EAC collects information on 2.508 patients. For this study, baseline characteristics of the patients referred in the semester following the COVID-19 lock-down (Jul-Dec 2020) were compared with: (i) patients referred in the semester immediately preceding the lock-down (Jul-Dec 2019); (ii) patients referred in the semester following the publication of the 2010 RA classification criteria (Jan-Jun 2011); (iii) patients referred in the semester preceding the publication of the 2010 criteria (Jul-Dec 2009).

Results: In the semester following the lock-down imposed by the COVID-19 pandemic, there was a decrease in the referral of patients with new-onset suspected IA compared with previous periods ( $n=71$ vs $n=91$ in the semester before the lock-down, $n=96$ in the first semester of 2011, $n=101$ in the second semester of 2009). Furthermore, fewer of the referred patients fulfilled RA criteria at presentation (36.6\% vs $44.3 \%, 46.5 \%$ and $42.9 \%$ in the other semesters). Among patients with RA, more were autoantibody-positive (72\% vs $50 \%, 49.1 \%$ and $52.2 \%)$. There was a trend for increased diagnostic delay in the overall cohort of RA after the COVID-19 lock-down (Figure 1A). The delay was particularly longer in autoantibody-positive patients, returning to the values seen before the introduction of the 2010 RA criteria (Figure 1B). In contrast, the few autoantibody-negative patients were referred earlier (Figure 1C). Disease activity at presentation was significantly higher in RA patients presenting after the lock-down compared with the progressive trend for reduction observed over the previous years, irrespective of the autoantibody status (Figure 1D-F). Such increase was determined by an inversion of the trend towards lower levels of objective parameters of inflammation, such as the swollen joint count (Figure 1G-I) and acute phase reactants, and a further increase in the secular trend towards worsening of patient-derived measures, such as the tender joint count and patient global assessment (Figure $1 \mathrm{~J}-\mathrm{L}$ ).
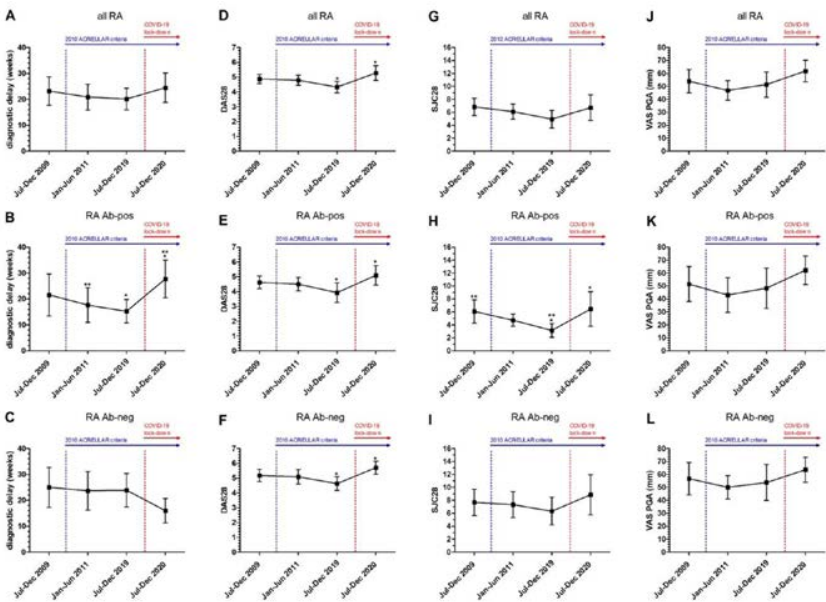

Figure 1. Effects of COVID-19 lock-down on new-onset RA at presentation.

Conclusion: The COVID-19 pandemic is posing unprecedented challenges in the management of patients suffering from chronic diseases. RA has returned to be diagnosed outside the window of opportunity, with a significantly higher inflammatory burden at presentation. The many benefits of early diagnosis, which have dramatically changed the outcomes of autoantibody-positive RA, are at risk of vanishing in short times. Equally important, autoantibody-negative RA is at risk of further under-diagnosis and under-treatment.

Disclosure of Interests: Bernardo D'Onofrio: None declared, Ludovico De Stefano: None declared, Bianca Lucia Palermo: None declared, Blerina Xoxi: None declared, Antonio Manzo: None declared, Carlomaurizio Montecucco Speakers bureau: BMS, Lilly, Sanofi, Pfizer, Galapagos, Roche, Novartis, Serena Bugatti Speakers bureau: BMS, Lilly, Sanofi, Pfizer, Galapagos

DOI: 10.1136/annrheumdis-2021-eular.3893

\section{POS1259 \\ FAVOURABLE SHORT-TERM COURSE OF COVID-19 IN PATIENTS WITH FAMILIAL MEDITERRANEAN FEVER USING BIOLOGIC AGENTS}

N. Aliyeva' ${ }^{1}$, B. Ç. Yalçın Dulundu', S. Amikishiyev ${ }^{1}$, S. Aghamuradov' ${ }^{1}$, M. Bektas ${ }^{1}$, B. Ince ${ }^{1}$, N. Koca ${ }^{1}$, Y. Yalçınkaya ${ }^{1}$, B. Artim-Esen ${ }^{1}$, M. Inanc ${ }^{1}$, A. Gül'. 'I'stanbul Medical Faculty, Rheumatology, İstanbul, Turkey

Background: COVID-19 runs a variable course resulting in acute respiratory distress syndrome and death in a subset of patients. The entry of SARS-CoV-2 into the cell stimulates innate immunity including NLRP3 inflammasome and lead to development of adaptive immunity later. Hyperinflammatory response with the release of proinflammatory cytokines including IL-1 $\beta$ and IL- 6 results in cytokine storm in some patients with a worse outcome. Colchicine acts on NLRP3 inflammasome and inhibits and IL-1 mediated inflammatory attacks in gout and familial Mediterranean fever (FMF) patients. Patients with inadequate response to colchicine may benefit from anti-IL-1 biologic agents such as anakinra and canakinumab. Recently, favourable effects of anakinra have been observed in COVID-19 patients with findings of cytokine storm.

Objectives: We aimed to evaluate the impact of COVID-19 among refractory FMF patients followed-up in tertiary referral with the treatment of biologic agents and also document the course of COVID-19 in these patients.

Methods: We searched out database of FMF patients to identify those using biologic agents (anti-IL-1, anti-IL-6 or anti-TNF) for colchicine-refractory FMF. We interviewed the patients using a standard questionnaire by phone call for symptomatic COVID-19 and evaluated those patients who described findings of COVID-19 further by their hospital records or inviting them to the hospital for additional investigations.

Results: We identified 183 patients and contacted 106 of them by phone in May-October 2020. A history of symptomatic COVID-19 was documented in 7 FMF patients who were on a biologic agent. Six were on anti-IL-1 and one was on anti-TNF, and one of the patients was not taking his biologic agents for 1 year. All of 7 patients had a favourable outcome. All but 1 patient followed at home and none of them developed findings of cytokine storm, thromboembolism and secondary bacterial infection. Hospitalized patient did not require intensive care unit (ICU) support or mechanical ventilation, and he was not given additional anti-inflammatory medications. 